\title{
Positive Solutions of a Third-Order Three-Point BVP with Sign-Changing Green's Function
}

\author{
Li-Juan Gao and Jian-Ping Sun \\ Department of Applied Mathematics, Lanzhou University of Technology, Lanzhou, Gansu 730050, China \\ Correspondence should be addressed to Jian-Ping Sun; jpsun2012@163.com
}

Received 3 April 2014; Revised 3 September 2014; Accepted 3 September 2014; Published 30 September 2014

Academic Editor: Zhen-Lai Han

Copyright (C) 2014 L.-J. Gao and J.-P. Sun. This is an open access article distributed under the Creative Commons Attribution License, which permits unrestricted use, distribution, and reproduction in any medium, provided the original work is properly cited.

We are concerned with the following third-order three-point boundary value problem: $u^{\prime \prime \prime}(t)=f(t, u(t)), t \in[0,1], u^{\prime}(0)=$ $u(1)=0$ and $u^{\prime \prime}(\eta)-\alpha u^{\prime}(1)=0$, where $\alpha \in[0,1)$ and $\eta \in[(14+\alpha) /(24-3 \alpha), 1)$. Although the corresponding Green's function is sign-changing, we still obtain the existence of at least two positive and decreasing solutions under some suitable conditions on $f$ by using the two-fixed-point theorem due to Avery and Henderson.

\section{Introduction}

Third-order differential equations arise from a variety of different areas of applied mathematics and physics, for example, in the deflection of a curved beam having a constant or varying cross-section, a three-layer beam, electromagnetic waves or gravity driven flows, and so on [1].

Recently, the existence of single or multiple positive solutions to some third-order three-point boundary value problems (BVPs for short) has received much attention from many authors; see [2-11] and the references therein. However, all the above-mentioned papers are achieved when the corresponding Green's functions are nonnegative, which is a very important condition.

In 2008, Palamides and Smyrlis [12] studied the existence of at least one positive solution to the singular third-order three-point BVP with an indefinitely signed Green's function:

$$
\begin{gathered}
u^{\prime \prime \prime}(t)=a(t) f(t, u(t)), \quad t \in(0,1), \\
u(0)=u(1)=u^{\prime \prime}(\eta)=0,
\end{gathered}
$$

where $\eta \in(17 / 24,1)$. Their technique was a combination of the Guo-Krasnoselskii fixed-point theorem and properties of the corresponding vector field.
Very recently, for the third-order three-point BVP with sign-changing Green's function

$$
\begin{gathered}
u^{\prime \prime \prime}(t)=f(t, u(t)), \quad t \in[0,1], \\
u^{\prime}(0)=u(1)=u^{\prime \prime}(\eta)=0,
\end{gathered}
$$

Sun and Zhao proved the existence of at least one or three positive solutions when $\eta \in(1 / 2,1)$ by using the GuoKrasnoselskii and Leggett-Williams fixed-point theorems in $[13,14]$ and obtained the existence of a positive solution when $\eta \in[2-\sqrt{2}, 1)$ by using iterative technique in [15].

In 2013, Li et al. [16] discussed the existence of a positive solution to the third-order three-point BVP with signchanging Green's function:

$$
\begin{gathered}
u^{\prime \prime \prime}(t)=f(t, u(t)), \quad t \in[0,1], \\
u^{\prime}(0)=u(1)=0, \quad u^{\prime \prime}(\eta)+\alpha u(0)=0,
\end{gathered}
$$

where $\alpha \in[0,2)$ and $\eta \in[(\sqrt{121+24 \alpha}-5) / 3(4+\alpha), 1)$. The main tool used was the Guo-Krasnoselskii fixed-point theorem.

It is worth mentioning that there are other types of works on sign-changing Green's functions which prove the existence of sign-changing solutions, positive in some cases; see Infante and Webb's papers [17-19]. 
Motivated greatly by the above-mentioned works, in this paper, we consider the following third-order three-point BVP:

$$
\begin{gathered}
u^{\prime \prime \prime}(t)=f(t, u(t)), \quad t \in[0,1], \\
u^{\prime}(0)=u(1)=0, \quad u^{\prime \prime}(\eta)-\alpha u^{\prime}(1)=0 .
\end{gathered}
$$

Throughout this paper, we always assume that $\alpha \in[0,1)$ and $\eta \in[(14+\alpha) /(24-3 \alpha), 1)$. Although the corresponding Green's function is sign-changing, we still obtain the existence of at least two positive and decreasing solutions under some suitable conditions on $f$. Our main tool is the wellknown Avery-Henderson two-fixed-point theorem.

To end this section, we state some fundamental definitions and the two-fixed-point theorem due to Avery and Henderson [20].

Let $K$ be a cone in a real Banach space $E$.

Definition 1. A functional $\psi: K \rightarrow R$ is said to be increasing on $K$ provided $\psi(x) \leq \psi(y)$ for all $x, y \in K$ with $x \leq y$, where $x \leq y$ if and only if $y-x \in K$.

Definition 2. Let $\gamma: K \rightarrow[0,+\infty)$ be continuous. For each $d>0$, we define the set

$$
K(\gamma, d)=\{u \in K: \gamma(u)<d\}
$$

Theorem 3. Let $\psi$ and $\gamma$ be increasing, nonnegative, and continuous functionals on $K$, and let $\omega$ be a nonnegative continuous functional on $K$ with $\omega(0)=0$ such that, for some $c>0$ and $M>0$,

$$
\gamma(u) \leq \omega(u) \leq \psi(u), \quad\|u\| \leq M \gamma(u)
$$

for all $u \in \overline{K(\gamma, c)}$. Suppose that there exist a completely continuous operator $T: \overline{K(\gamma, c)} \rightarrow K$ and $0<a<b<c$ such that

$$
\omega(\lambda u) \leq \lambda \omega(u) \quad \text { for } 0 \leq \lambda \leq 1, u \in \partial K(\omega, b),
$$

and
(1) $\gamma(T u)>c$ for all $u \in \partial K(\gamma, c)$;
(2) $\omega(T u)<b$ for all $u \in \partial K(\omega, b)$;
(3) $K(\psi, a) \neq \emptyset$, and $\psi(T u)>$ a for all $u \in \partial K(\psi, a)$.

Then $T$ has at least two fixed points $u_{1}$ and $u_{2}$ in $\overline{K(\gamma, c)}$ such that

$$
\begin{aligned}
& a<\psi\left(u_{1}\right) \quad \text { with } \omega\left(u_{1}\right)<b, \\
& b<\omega\left(u_{2}\right) \quad \text { with } \gamma\left(u_{2}\right)<c .
\end{aligned}
$$

\section{Preliminaries}

For the BVP

$$
\begin{gathered}
u^{\prime \prime \prime}(t)=0, \quad t \in[0,1], \\
u^{\prime}(0)=u(1)=0, \quad u^{\prime \prime}(\eta)-\alpha u^{\prime}(1)=0,
\end{gathered}
$$

we have the following lemma.
Lemma 4. The BVP (9) has only trivial solution.

Proof. It is simple to check.

Now, for any $y \in C[0,1]$, we consider the BVP

$$
\begin{gathered}
u^{\prime \prime \prime}(t)=y(t), \quad t \in[0,1], \\
u^{\prime}(0)=u(1)=0, \quad u^{\prime \prime}(\eta)-\alpha u^{\prime}(1)=0 .
\end{gathered}
$$

After a direct computation, one may obtain the expression of Green's function $G(t, s)$ of the BVP $(10)$ as follows:

$$
G(t, s)=g(t, s)+k(t, s)+ \begin{cases}0, & s \geq \eta \\ \frac{1-t^{2}}{2(1-\alpha)}, & s<\eta\end{cases}
$$

where

$$
\begin{gathered}
g(t, s)=-\frac{\alpha\left(1-t^{2}\right)(1-s)}{2(1-\alpha)}-\frac{(1-s)^{2}}{2}, \\
(t, s) \in[0,1] \times[0,1], \\
k(t, s)= \begin{cases}0, & 0 \leq t \leq s \leq 1, \\
\frac{(t-s)^{2}}{2}, & 0 \leq s \leq t \leq 1 .\end{cases}
\end{gathered}
$$

It is not difficult to verify that the $G(t, s)$ has the following properties:

$$
\begin{array}{ll}
G(t, s) \geq 0 & \text { for } 0 \leq s<\eta \\
G(t, s) \leq 0 & \text { for } \eta \leq s \leq 1 .
\end{array}
$$

Moreover, for $s \geq \eta$,

$$
\begin{aligned}
\max \{G(t, s): t \in[0,1]\} & =G(1, s)=0, \\
\min \{G(t, s): t \in[0,1]\} & =G(0, s) \\
& =-\frac{\alpha(1-s)}{2(1-\alpha)}-\frac{(1-s)^{2}}{2}
\end{aligned}
$$

and for $s<\eta$,

$$
\begin{aligned}
& \max \{G(t, s): t \in[0,1]\}=G(0, s) \\
& \qquad=\frac{1-\alpha(1-s)}{2(1-\alpha)}-\frac{(1-s)^{2}}{2}, \\
& \text { Let } \min \{G(t, s): t \in[0,1]\}=G(1, s)=0 . \\
& K_{0}=\{y \in C[0,1]: y(t) \text { is nonnegative } \\
& \text { and decreasing on }[0,1]\} .
\end{aligned}
$$

Then $K_{0}$ is a cone in $C[0,1]$.

Lemma 5. Let $y \in K_{0}$ and $u(t)=\int_{0}^{1} G(t, s) y(s) d s, t \in[0,1]$. Then $u$ is the unique solution of the BVP (10) and $u \in K_{0}$. Moreover, $u(t)$ is concave on $[0, \eta]$. 
Proof. For $t \in[0, \eta]$, we have

$$
\begin{aligned}
u(t)= & \int_{0}^{t}\left[g(t, s)+\frac{(t-s)^{2}}{2}+\frac{1-t^{2}}{2(1-\alpha)}\right] y(s) d s \\
& +\int_{t}^{\eta}\left[g(t, s)+\frac{1-t^{2}}{2(1-\alpha)}\right] y(s) d s \\
& +\int_{\eta}^{1} g(t, s) y(s) d s .
\end{aligned}
$$

Since $y \in K_{0}$ and $\eta \geq(14+\alpha) /(24-3 \alpha)$ implies that $\eta>$ $\alpha /(1+\alpha)$, we get

$$
\begin{aligned}
u^{\prime}(t)= & -\frac{\alpha t}{1-\alpha} \int_{0}^{\eta} s y(s) d s-\int_{0}^{t} s y(s) d s \\
& -t \int_{t}^{\eta} y(s) d s+\frac{\alpha t}{1-\alpha} \int_{\eta}^{1}(1-s) y(s) d s \\
\leq & y(\eta)\left[-\frac{\alpha t}{1-\alpha} \int_{0}^{\eta} s d s-\int_{0}^{t} s d s\right. \\
& \left.-t \int_{t}^{\eta} d s+\frac{\alpha t}{1-\alpha} \int_{\eta}^{1}(1-s) d s\right] \\
= & t y(\eta)\left[\frac{\alpha(1-2 \eta)}{2(1-\alpha)}-\eta+\frac{t}{2}\right] \\
\leq & t y(\eta)\left[\frac{\alpha(1-2 \eta)}{2(1-\alpha)}-\frac{\eta}{2}\right] \\
\leq & t \in[0, \eta] .
\end{aligned}
$$

At the same time, $y \in K_{0}$ and $\eta \geq(14+\alpha) /(24-3 \alpha)>1 / 2$ shows that

$$
\begin{aligned}
u^{\prime \prime}(t)= & -\frac{\alpha}{1-\alpha} \int_{0}^{\eta} s y(s) d s-\int_{t}^{\eta} y(s) d s \\
& +\frac{\alpha}{1-\alpha} \int_{\eta}^{1}(1-s) y(s) d s \\
\leq & y(\eta)\left[-\frac{\alpha}{1-\alpha} \int_{0}^{\eta} s d s-\int_{t}^{\eta} d s+\frac{\alpha}{1-\alpha} \int_{\eta}^{1}(1-s) d s\right] \\
= & y(\eta)\left[\frac{\alpha(1-2 \eta)}{2(1-\alpha)}-\eta+t\right] \\
\leq & \frac{y(\eta) \alpha(1-2 \eta)}{2(1-\alpha)} \\
\leq & 0, \quad t \in[0, \eta] .
\end{aligned}
$$

For $t \in(\eta, 1]$, we have

$$
\begin{aligned}
u(t)= & \int_{0}^{\eta}\left[g(t, s)+\frac{(t-s)^{2}}{2}+\frac{1-t^{2}}{2(1-\alpha)}\right] y(s) d s \\
& +\int_{\eta}^{t}\left[g(t, s)+\frac{(t-s)^{2}}{2}\right] y(s) d s \\
& +\int_{t}^{1} g(t, s) y(s) d s .
\end{aligned}
$$

In view of $y \in K_{0}$ and $\eta>1 / 2$, we get

$$
\begin{aligned}
u^{\prime}(t)= & -\frac{\alpha t}{1-\alpha} \int_{0}^{\eta} s y(s) d s+\int_{\eta}^{t}(t-s) y(s) d s \\
& -\int_{0}^{\eta} s y(s) d s+\frac{\alpha t}{1-\alpha} \int_{\eta}^{1}(1-s) y(s) d s \\
\leq & y(\eta)\left[-\frac{\alpha t}{1-\alpha} \int_{0}^{\eta} s d s+\int_{\eta}^{t}(t-s) d s\right. \\
& \left.-\int_{0}^{\eta} s d s+\frac{\alpha t}{1-\alpha} \int_{\eta}^{1}(1-s) d s\right] \\
= & t y(\eta)\left[\frac{\alpha(1-2 \eta)}{2(1-\alpha)}+\frac{t-2 \eta}{2}\right] \\
\leq & t y(\eta)\left[\frac{1-2 \eta}{2(1-\alpha)}\right] \\
\leq & 0, \quad t \in(\eta, 1] .
\end{aligned}
$$

Obviously, $u^{\prime \prime \prime}(t)=y(t)$ for $t \in[0,1], u^{\prime}(0)=u(1)=$ 0 , and $u^{\prime \prime}(\eta)-\alpha u^{\prime}(1)=0$. This shows that $u$ is a solution of the BVP (10). The uniqueness follows immediately from Lemma 4. Since $u^{\prime}(t) \leq 0$ for $t \in[0,1]$ and $u(1)=0$, we have $u(t) \geq 0$ for $t \in[0,1]$. So, $u \in K_{0}$. In view of $u^{\prime \prime}(t) \leq 0$ for $t \in[0, \eta]$, we know that $u(t)$ is concave on $[0, \eta]$.

Throughout this paper, for any $u \in C[0,1]$, we define $\|u\|=\max _{t \in[0,1]}|u(t)|$.

Lemma 6. Let $y \in K_{0}$. Then the unique solution $u$ of the BVP (10) satisfies

$$
\min _{t \in[0, \theta]} u(t) \geq \theta^{*}\|u\|
$$

where $\theta \in(0,1 / 2]$ and $\theta^{*}=(\eta-\theta) / \eta$.

Proof. By Lemma 5, we know that $u(t)$ is concave on $[0, \eta]$; thus, for $t \in[0, \eta]$,

$$
u(t) \geq \frac{\eta-t}{\eta} u(0)+\frac{t}{\eta} u(\eta)
$$

At the same time, it follows from $u \in K_{0}$ that $\|u\|=u(0)$, which together with (23) implies that

$$
u(t) \geq \frac{\eta-t}{\eta}\|u\|, \quad t \in[0, \eta] .
$$


Consequently,

$$
\min _{t \in[0, \theta]} u(t)=u(\theta) \geq \frac{\eta-\theta}{\eta}\|u\|=\theta^{*}\|u\| .
$$

\section{Main Results}

In the remainder of this paper, we always assume that $f$ : $[0,1] \times[0,+\infty) \rightarrow[0,+\infty)$ is continuous and satisfies the following two conditions:

(H1) for each $u \in[0,+\infty)$, the mapping $t \mapsto f(t, u)$ is decreasing;

(H2) for each $t \in[0,1]$, the mapping $u \mapsto f(t, u)$ is increasing.

Let

$$
K=\left\{u \in K_{0}: \min _{t \in[0, \theta]} u(t) \geq \theta^{*}\|u\|\right\} .
$$

Then it is easy to see that $K$ is a cone in $C[0,1]$.

Now, we define an operator $T$ as follows:

$$
(T u)(t)=\int_{0}^{1} G(t, s) f(s, u(s)) d s, \quad u \in K, t \in[0,1] .
$$

First, it is obvious that if $u$ is a fixed point of $T$ in $K$, then $u$ is a nonnegative and decreasing solution of the BVP (4). Next, by Lemmas 5 and 6, we know that $T: K \rightarrow K$. Furthermore, although $G(t, s)$ is not continuous, it follows from known textbook results, for example, see [21], that $T: K \rightarrow K$ is completely continuous.

For convenience, we denote

$$
A=\int_{0}^{\theta} G(\eta, s) d s, \quad B=\int_{0}^{\eta} G(\theta, s) d s .
$$

Theorem 7. Suppose that there exist numbers $a, b$, and $c$ with $0<a<b<\theta^{*} c$ such that

$$
\begin{gathered}
f(\theta, c)>\frac{c}{A}, \\
f\left(0, \frac{b}{\theta^{*}}\right)<\frac{b}{B}, \\
f\left(\theta, \theta^{*} a\right)>\frac{a}{A} .
\end{gathered}
$$

Then the BVP (4) has at least two positive and decreasing solutions.

Proof. First, we define the increasing, nonnegative, and continuous functionals $\gamma, \omega$, and $\psi$ on $K$ as follows:

$$
\begin{aligned}
& \gamma(u)=\min _{t \in[0, \theta]} u(t)=u(\theta), \\
& \omega(u)=\max _{t \in[\theta, 1]} u(t)=u(\theta), \\
& \psi(u)=\max _{t \in[0,1]} u(t)=u(0) .
\end{aligned}
$$

Obviously, for any $u \in K, \gamma(u)=\omega(u) \leq \psi(u)$. At the same time, for each $u \in K$, in view of $\gamma(u)=\min _{t \in[0, \theta]} u(t) \geq \theta^{*}\|u\|$, we have

$$
\|u\| \leq \frac{1}{\theta^{*}} \gamma(u) \quad \text { for } u \in K .
$$

In addition, we also note that

$$
\omega(\lambda u)=\lambda \omega(u) \quad \text { for } 0 \leq \lambda \leq 1, u \in K
$$

Next, for any $u \in K$, we claim that

$$
\int_{\theta}^{1} G(\eta, s) f(s, u(s)) d s \geq 0
$$

In fact, it follows from $(H 1),(H 2)$, and $\eta \geq(14+\alpha) /(24-3 \alpha)$ that

$$
\begin{aligned}
& \int_{\theta}^{1} G(\eta, s) f(s, u(s)) d s \\
& =\int_{\theta}^{\eta} G(\eta, s) f(s, u(s)) d s+\int_{\eta}^{1} G(\eta, s) f(s, u(s)) d s \\
& \geq f(\eta, u(\eta))\left[\int_{\theta}^{\eta} G(\eta, s) d s+\int_{\eta}^{1} G(\eta, s) d s\right] \\
& =f(\eta, u(\eta)) \\
& \times\left[\int_{\theta}^{\eta}\left(g(\eta, s)+\frac{(\eta-s)^{2}}{2}+\frac{1-\eta^{2}}{2(1-\alpha)}\right) d s\right. \\
& \left.+\int_{\eta}^{1} g(\eta, s) d s\right] \\
& =\frac{(1-\eta) f(\eta, u(\eta))}{12(1-\alpha)} \\
& \times\left[(4+2 \alpha) \eta^{2}+(4-\alpha) \eta-\alpha-2+(3 \alpha-3 \alpha \eta-6) \theta^{2}\right] \\
& \geq \frac{(1-\eta) f(\eta, u(\eta))}{12(1-\alpha)} \\
& \times\left[(4+2 \alpha) \eta^{2}+\left(4-\frac{7}{4} \alpha\right) \eta-\frac{1}{4} \alpha-\frac{7}{2}\right] \\
& \geq \frac{(1-\eta) f(\eta, u(\eta))}{12(1-\alpha)}\left[\left(6-\frac{3}{4} \alpha\right) \eta-\frac{1}{4} \alpha-\frac{7}{2}\right] \\
& \geq 0 \text {. }
\end{aligned}
$$

Now, we assert that $\gamma(T u)>c$ for all $u \in \partial K(\gamma, c)$.

To prove this, let $u \in \partial K(\gamma, c)$; that is, $u \in K$ and $\gamma(u)=$ $u(\theta)=c$. Then

$$
u(t) \geq u(\theta)=c, \quad t \in[0, \theta] .
$$


Since $(T u)(t)$ is decreasing on [0,1], it follows from (29), (35), (37), (H1), and (H2) that

$$
\begin{aligned}
\gamma(T u) & =(T u)(\theta) \\
& \geq(T u)(\eta) \\
& =\int_{0}^{1} G(\eta, s) f(s, u(s)) d s \\
& \geq \int_{0}^{\theta} G(\eta, s) f(s, u(s)) d s \\
& \geq \int_{0}^{\theta} G(\eta, s) f(\theta, c) d s \\
& >\frac{c}{A} \int_{0}^{\theta} G(\eta, s) d s \\
& =c .
\end{aligned}
$$

Then, we assert that $\omega(T u)<b$ for all $u \in \partial K(\omega, b)$.

To see this, suppose that $u \in \partial K(\omega, b)$; that is, $u \in K$ and $\omega(u)=b$. Since $\|u\| \leq\left(1 / \theta^{*}\right) \gamma(u)=\left(1 / \theta^{*}\right) \omega(u)$, we have

$$
0 \leq u(t) \leq\|u\| \leq \frac{b}{\theta^{*}}, \quad t \in[0, \eta]
$$

In view of the properties of $G(t, s),(30),(39),(H 1)$, and (H2), we get

$$
\begin{aligned}
\omega(T u) & =(T u)(\theta) \\
& =\int_{0}^{1} G(\theta, s) f(s, u(s)) d s \\
& \leq \int_{0}^{\eta} G(\theta, s) f(s, u(s)) d s \\
& \leq \int_{0}^{\eta} G(\theta, s) f\left(0, \frac{b}{\theta^{*}}\right) d s \\
& <\frac{b}{B} \int_{0}^{\eta} G(\theta, s) d s \\
& =b .
\end{aligned}
$$

Finally, we assert that $K(\psi, a) \neq \emptyset$ and $\psi(T u)>a$ for all $u \in \partial K(\psi, a)$.

In fact, the constant function $a / 2 \in K(\psi, a)$. Moreover, for $u \in \partial K(\psi, a)$, that is, $u \in K$ and $\psi(u)=u(0)=a$, we know that

$$
u(t) \geq \theta^{*}\|u\|=\theta^{*} u(0)=\theta^{*} a, \quad t \in[0, \theta] .
$$

Since $(T u)(t)$ is decreasing on $[0,1]$, it follows from (31), (35), (41), (H1), and $(H 2)$ that

$$
\begin{aligned}
\psi(T u) & =(T u)(0) \\
& \geq(T u)(\eta) \\
& =\int_{0}^{1} G(\eta, s) f(s, u(s)) d s \\
& \geq \int_{0}^{\theta} G(\eta, s) f(s, u(s)) d s \\
& \geq \int_{0}^{\theta} G(\eta, s) f\left(\theta, \theta^{*} a\right) d s \\
& >\frac{a}{A} \int_{0}^{\theta} G(\eta, s) d s \\
& =a .
\end{aligned}
$$

To sum up, all the hypotheses of Theorem 3 are satisfied. Hence, $T$ has at least two fixed points $u_{1}$ and $u_{2}$; that is, the BVP (4) has at least two positive and decreasing solutions $u_{1}$ and $u_{2}$ satisfying

$$
\begin{aligned}
& a<\max _{t \in[0,1]} u_{1}(t) \quad \text { with } \max _{t \in[\theta, 1]} u_{1}(t)<b, \\
& b<\max _{t \in[\theta, 1]} u_{2}(t) \quad \text { with } \min _{t \in[0, \theta]} u_{2}(t)<c .
\end{aligned}
$$

\section{Conflict of Interests}

The authors declare that there is no conflict of interests regarding the publication of this paper.

\section{References}

[1] M. Greguš, Third Order Linear Differential Equations, vol. 22, Reidel, Dordrecht, The Netherlands, 1987.

[2] D. R. Anderson, "Green's function for a third-order generalized right focal problem," Journal of Mathematical Analysis and Applications, vol. 288, no. 1, pp. 1-14, 2003.

[3] Z. Bai and X. Fei, "Existence of triple positive solutions for a third order generalized right focal problem," Mathematical Inequalities and Applications, vol. 9, no. 3, pp. 437-444, 2006.

[4] X. Feng, H. Feng, and D. Bai, "Eigenvalue for a singular thirdorder three-point boundary value problem," Applied Mathematics and Computation, vol. 219, no. 18, pp. 9783-9790, 2013.

[5] L.-J. Guo, J.-P. Sun, and Y.-H. Zhao, "Existence of positive solutions for nonlinear third-order three-point boundary value problems," Nonlinear Analysis: Theory, Methods \& Applications, vol. 68, no. 10, pp. 3151-3158, 2008.

[6] A. P. Palamides and N. M. Stavrakakis, "Existence and uniqueness of a positive solution for a third-order three-point boundary-value problem," Electronic Journal of Differential Equations, vol. 2010, no. 155, pp. 1-12, 2010.

[7] Y. Sun, "Positive solutions of singular third-order three-point boundary value problem," Journal of Mathematical Analysis and Applications, vol. 306, no. 2, pp. 589-603, 2005. 
[8] Y. Sun, "Positive solutions for third-order three-point nonhomogeneous boundary value problems," Applied Mathematics Letters, vol. 22, no. 1, pp. 45-51, 2009.

[9] F. J. Torres, "Positive solutions for a third-order three-point boundary-value problem," Electronic Journal of Differential Equations, vol. 2013, no. 147, pp. 1-11, 2013.

[10] Q. Yao, "The existence and multiplicity of positive solutions for a third-order three-point boundary value problem," Acta Mathematicae Applicatae Sinica, vol. 19, no. 1, pp. 117-122, 2003.

[11] Q. Yao, "Positive solutions of singular third-order three-point boundary value problems," Journal of Mathematical Analysis and Applications, vol. 354, no. 1, pp. 207-212, 2009.

[12] A. P. Palamides and G. Smyrlis, "Positive solutions to a singular third-order three-point boundary value problem with an indefinitely signed Green's function," Nonlinear Analysis: Theory, Methods and Applications, vol. 68, no. 7, pp. 2104-2118, 2008.

[13] J.-P. Sun and J. Zhao, "Positive solution for a third-order threepoint boundary value problem with sign-changing Green's function," Communications in Applied Analysis, vol. 16, no. 2, pp. 219-228, 2012.

[14] J.-P. Sun and J. Zhao, "Multiple positive solutions for a thirdorder three-point BVP with sign-changing Green's function," Electronic Journal of Differential Equations, vol. 2012, no. 118, pp. $1-7,2012$.

[15] J.-P. Sun and J. Zhao, "Iterative technique for a third-order three-point BVP with sign-changing Green's function," Electronic Journal of Differential Equations, vol. 2013, no. 215, pp. 1-9, 2013.

[16] X.-L. Li, J.-P. Sun, and F.-D. Kong, "Existence of positive solution for a third-order three-point BVP with sign-changing Green's function," Electronic Journal of Qualitative Theory of Differential Equations, vol. 2013, no. 30, pp. 1-11, 2013.

[17] G. Infante and J. R. L. Webb, "Nonzero solutions of Hammerstein integral equations with discontinuous kernels," Journal of Mathematical Analysis and Applications, vol. 272, no. 1, pp. 3042, 2002.

[18] G. Infante and J. R. L. Webb, "Three-point boundary value problems with solutions that change sign," Journal of Integral Equations and Applications, vol. 15, no. 1, pp. 37-57, 2003.

[19] G. Infante and J. R. L. Webb, "Loss of positivity in a nonlinear scalar heat equation," NoDEA: Nonlinear Differential Equations and Applications, vol. 13, no. 2, pp. 249-261, 2006.

[20] R. I. Avery and J. Henderson, "Two positive fixed points of nonlinear operators on ordered Banach spaces," Communications on Applied Nonlinear Analysis, vol. 8, no. 1, pp. 27-36, 2001.

[21] R. H. Martin, Nonlinear Operators and Differential Equations in Banach Spaces, John Wiley \& Sons, New York, NY, USA, 1976. 


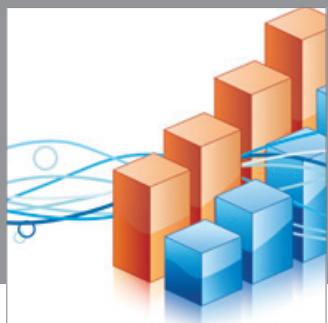

Advances in

Operations Research

mansans

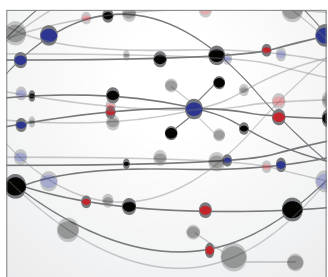

The Scientific World Journal
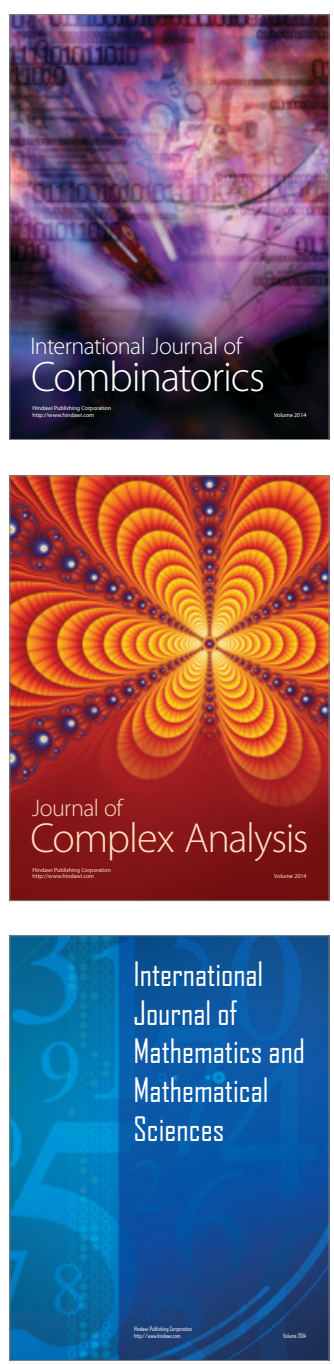
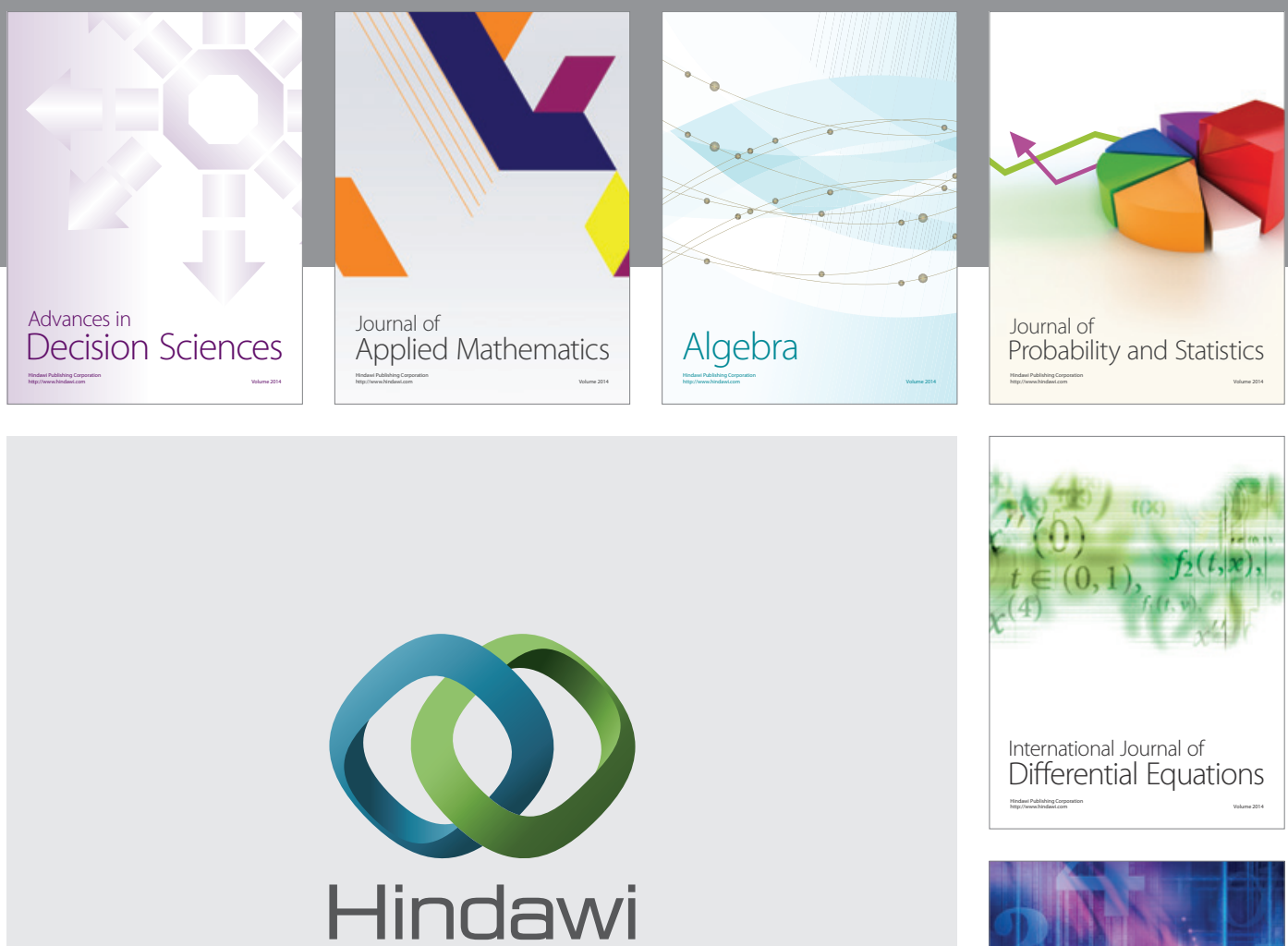

Submit your manuscripts at http://www.hindawi.com
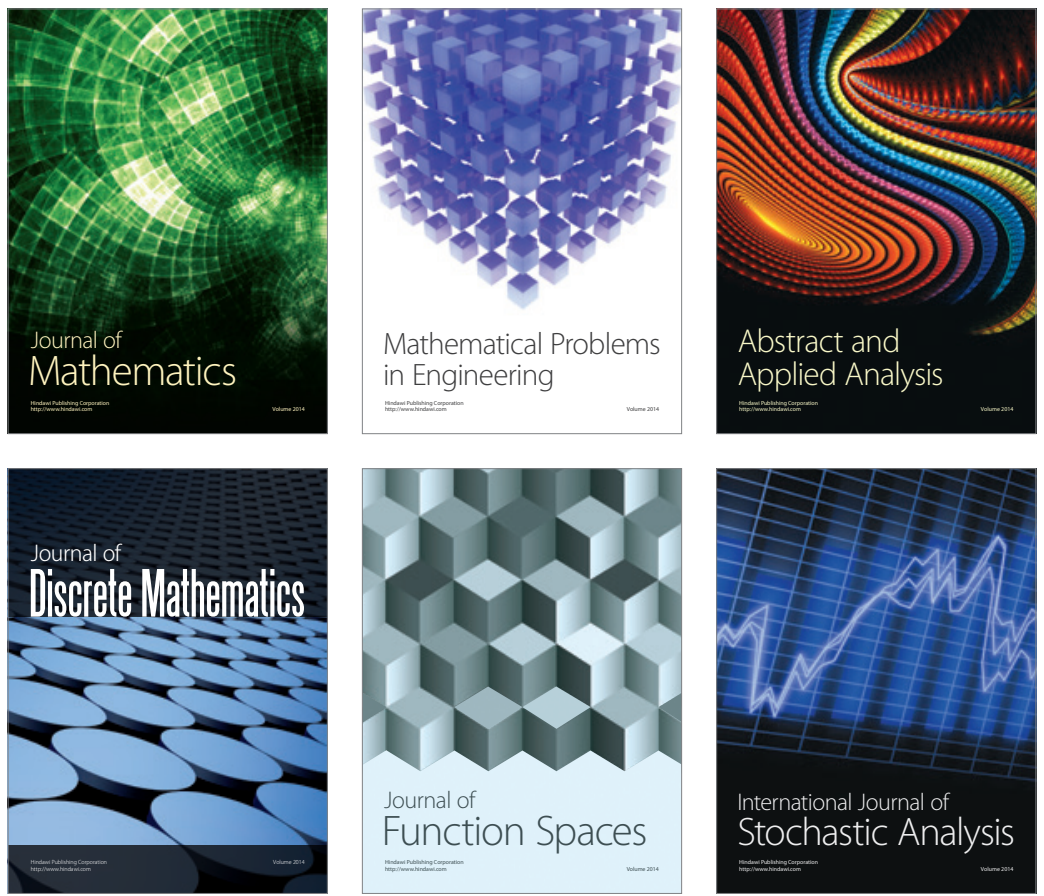

Journal of

Function Spaces

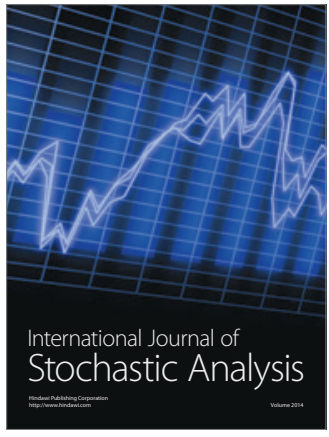

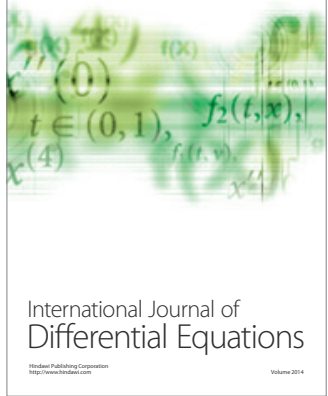
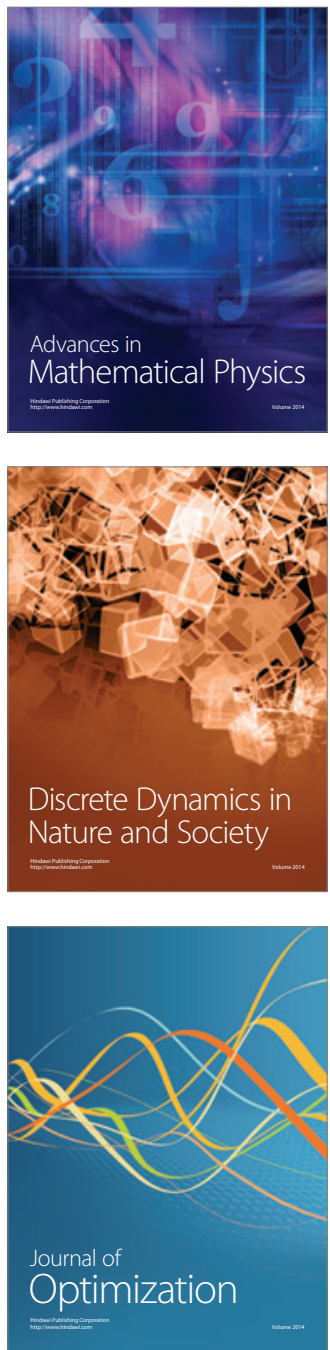